\title{
HES 1 is essential for chemoresistance induced by stellate cells and is associated with poor prognosis in pancreatic cancer
}

\author{
FENG CAO, JIA LI, HAICHEN SUN, SHUANG LIU, YEQING CUI and FEI LI \\ Department of General Surgery, Xuanwu Hospital, Capital Medical University, Beijing 100053, P.R. China
}

Received October 20, 2014; Accepted January 7, 2015

DOI: 10.3892/or.2015.3789

\begin{abstract}
The role of pancreatic stellate cells (PSCs) has been established in many studies. However, the potential mechanism for the chemoresistance induced by PSCs has not been fully elucidated. In the present study, human pancreatic cancer cell lines were directly or indirectly co-cultured with PSCs. The inhibition rate and $\mathrm{IC}_{50}$ values were assessed to determine the ability of chemoresistance. RT-PCR and western blot analysis were used to evaluate Hes 1 and Jagged 1 expression before and after co-culture with PSCs. To determine the relationship between Hes 1 expression and survival in pancreatic cancer patients, Kaplan-Meier survival analysis was performed. PSCs promoted the expression of Hes 1 in both PANC-1 and BxPC-3 cell lines and induced chemoresistance to gemcitabine. A Notch signaling pathway inhibitor (L1790) and Hes 1 siRNA reversed the chemoresistance induced by PSCs. In 72 resected pancreatic cancer patients, high Hes 1 expression was observed in 34 patients with shorter overall and progressionfree survival times. In conclusion, Hes 1 is essential for chemoresistance induced by PSCs and is associated with poor prognosis in pancreatic cancer patients. Therapy targeting the Notch signaling pathway may reverse chemoresistance and improve survival in patients with pancreatic cancer.
\end{abstract}

\section{Introduction}

Despite rapid advances in diagnostic and surgical procedures in the past decade, pancreatic cancer remains the most lethal human malignancy with an extremely low 5-year survival rate (1-3). In the USA, in 2014, it was estimated that there were 46,420 newly diagnosed patients and 39,590 died of this disease (4). A low radical resection rate and insensitive to chemoradiotherapy are the main reasons for the short survival time (1,5-9). Further insights into the mechanisms causing

Correspondence to: Professor Fei Li, Department of General Surgery, Xuanwu Hospital, Capital Medical University, Beijing 100053, P.R. China

E-mail: feili36@ccmu.edu.cn

Key words: pancreatic cancer, chemoresistance, Hes 1, gemcitabine, pancreatic stellate cells primary or secondary chemoresistance are urgently needed and may reveal new prospects for therapy.

Pancreatic stellate cells (PSCs), first isolated and cultured by Bachem et al and Apte et al in 1998, are the main source of pancreatic fibrosis in patients with chronic pancreatitis and pancreatic cencer $(10,11)$. Many studies have demonstrated that PSCs promote the progression of pancreatic cancer including cell proliferation, migration, invasion and even distant metastasis (12-16). However, the role of PSCs in the chemoresistance of pancreatic cancer has not been fully elucidated.

As an ancient cell signaling system, Notch plays a key role in organ development, cell fate determination and stem cell maintenance $(17,18)$. In adults, alteration of these functions has been associated with many types of malignancies including pancreatic cancer (19). A recent study demonstrated that Notch components, Notch-1, -3 and -4, HES-1 and HEY-1 presented significantly higher nuclear expression in locally advanced and metastatic tumors compared to resectable cancers. In survival analyses, nuclear Notch-3 and HEY-1 expression levels were significantly associated with reduced overall and disease-free survival following curative intent surgery therapy (20). Targeting the Notch signaling pathway for pancreatic cancer showed promising results in preclinical studies (21-24). The present study revealed that PSCs promoted expression of the Notch component, Hes 1 and chemoresistance to gemcitabine in pancreatic cancer. The Notch signaling pathway inhibitor (L1790) and Hes 1 siRNA reversed the effect of PSCs on chemoresistance. In clinical study, we found that HES 1 expression was associated with shorter overall and diseasefree survival in pancreatic cancer patients.

\section{Materials and methods}

PSC isolation and cell culture. PSCs were isolated from the normal rat pancreas according to the method established by Apte et al (11) and were maintained in Dulbecco's modified Eagle's medium (DMEM) containing 10\% fetal bovine serum (FBS). Two human pancreatic cancer cell lines (PANC-1 and BxPC-3) were cultured in DMEM supplemented with $10 \%$ FBS, L-glutamine, and $1 \%$ penicillin and streptomycin in a $5 \% \mathrm{CO}_{2}$ atmosphere at $37^{\circ} \mathrm{C}$ unless otherwise indicated.

Co-culture of pancreatic cancer and stellate cells. Pancreatic cancer cells (PANC-1, $1.5 \times 10^{5}$ cells/well and BxPC-3, $1.0 \times 10^{5}$ cells/well) were seeded in 6 -well culture plates 
(Corning Costar, NY, USA). PSCs $\left(3 \times 10^{5}\right.$ cells/culture insert) were seeded into the culture inserts of $1.0 \mu \mathrm{m}$ pore size (Corning Costar). On the following day, the culture insets seeded with PSCs were placed into the 6-well plates containing pancreatic cancer cells, and incubation was continued up to 3 days in DMEM supplemented with $1 \%$ FBS, penicillin and streptomycin. Cancer cells after co-culture were collected for chemoresistance analysis.

Preparation of PSC conditioned medium. PSC conditioned medium was prepared according to the method as described by Hwang et al (14). Briefly, when PSCs were grown to 70 to $80 \%$ confluence in $20-\mathrm{cm}^{2}$ dishes in DMEM/10\% FCS, the medium was replaced with serum-free DMEM, and the cells were cultured for $48 \mathrm{~h}$. Then the medium was collected, centrifuged and the supernatant was concentrated with Centricon YM-3 filters (Millipore Corp., Billerica, MA, USA).

Gemcitabine treatment. To explore the effect of PSCs on chemoresistance, the cancer cells (co-cultured or not co-cultured with PSCs) were seeded in 6-well (PANC-1, $1.5 \times 10^{5}$ cells/well and BxPC-3, 1.0x $10^{5}$ cells/well) or 96-well plates $\left(4 \times 10^{3}\right.$ and $3 \times 10^{3}$ cells/well). Cancer cells cultured with PSCs were incubated with PSC conditional medium. The Cell Counting Kit-8 (CCK-8) was used to calculate the inhibitory rate after incubation with gemcitabine $(100 \mathrm{ng} / \mathrm{ml}$; SigmaAldrich, St. Louis, MO, USA) for $48 \mathrm{~h}$. To assess the $\mathrm{IC}_{50}$ value, different concentrations of gemcitabine $(100,10,1,0.1$ and $0.01 \mathrm{mg} / \mathrm{ml} ; 1,0.1$, and $0.01 \mu \mathrm{g} / \mathrm{ml}$; and 1 and $0.1 \mathrm{ng} / \mathrm{ml}$ ) were added to the co-culture system. GraphPad Prism 6 was used to calculate the $\mathrm{IC}_{50}$ value.

Measurement of apoptosis. Annexin V-FITC/PI (BD Pharmingen, San Diego, CA, USA) was used for detecting apoptotic cells according to the manufacturer's instructions. Briefly, cells were washed, trypsinized, centrifuged and then resuspended at $1 \times 10^{6}$ cells $/ \mathrm{ml}$, and then incubated in binding buffer containing Annexin V-FITC (5 ml) and PI (10 ml) for $15 \mathrm{~min}$ in the dark. A BD flow cytometer was used for analysis.

Hes 1 siRNA transfection. Pancreatic cancer cells were transfected with Hes 1 siRNA (sense, 5'-AAAGAUAGCUC CCGGCAUU-3') using Lipofectamine 2000 according to the manufacturer's instructions.

RNA isolation, $c D N A$ synthesis and real-time reverse transcription-PCR. The total RNA from pancreatic cancer cells and siRNA-transfected cancer cells was isolated with TRIzol (Invitrogen Life Technologies, Carlsbad, CA, USA) and purified with the RNeasy Mini kit and RNase-free DNase set (Qiagen, Hilden, Germany) according to the manufacturer's protocols. Total RNA was reverse transcribed using the High Capacity cDNA reverse transcription kit (Applied Biosystems, Foster City, CA, USA) and then mRNA expression was quantified using the TaqMan Gene Expression Assay (Applied Biosystems). The primers used in the PCR reaction for Hes 1 and Jagged-1 were: Hes 1 forward, 5'-GGGCAAGAATAAAT GAAAG-3' and reverse, 5'-GCGCGGTACTTCCCCAA CAC-3' and Jagged-1 forward, 5'-GGGCCAGACTGCAGGA
TAAAC-3' and reverse, 5'-CGCCGTGCCCTTTGTGGAG-3', respectively.

Western blot analysis. To detect changes in the protein levels of Hes 1 (sc-13844) and Jagged 1 (sc-6011) (both from Santa Cruz Biotechnology, Santa Cruz, CA, USA), standard western immunoblotting techniques were used. Cells were lysed in lysis buffer by incubating for $20 \mathrm{~min}$ at $4^{\circ} \mathrm{C}$. Total proteins were fractionated using SDS-PAGE and transferred onto nitrocellulose membrane for western blotting in routine manner. The blots were then detected using ECL (Illumina, Inc., San Diego, CA, USA).

Tissue specimens and immunohistochemistry. Human specimens from 72 patients with pancreatic cancer who underwent R0 resection between January 2004 and 2013 were obtained from the Tissue Bank of the Department of General Surgery, Xuanwu Hospital, Beijing, China. The study was approved by the Ethics Committee of the hospital. Immunohistochemistry on formalin-fixed, paraffin-embedded samples was conducted as previously described. The slides were graded into 3 categories as described earlier (25), from grade 1 to 3 , as follows: grade 1, 0-25\% staining; grade 2, 26-50\% staining; and grade $3,>50 \%$ staining.

Statistical analyses. Experiments presented in the figures are representative of at least 3 repetitions. Continuous data are presented as mean $\pm \mathrm{SE}$ and were analyzed by the two-tailed Student's t-test. Categorical variables were analyzed using Chi-square tests. Survival was assessed according to the Kaplan-Meier method; the survival differences were analyzed using the log-rank test. Univariate and multivariate survival analyses were performed using Cox proportional hazard model. Results are reported as relative risk (RR) and 95\% confidence intervals (95\% CI). SPSS software (version 18.0; SPSS. Inc., Chicago, IL, USA) was used for analysis with a significance level of $\mathrm{P}<0.05$.

\section{Results}

PSC isolation. As described previously, PSCs are mainly located in the interstitium between acini, and negative staining for $\alpha$-smooth muscle actin ( $\alpha$-SMA) is noted in quiescent PSCs. However, we found that active PSCs were also occasionally visible in the islet in the normal rat pancreas (Fig. 1A). After isolation, Oil-red O staining and vitamin A autofluorescence showed the droplets in the cytoplasm in quiescent PSCs (Fig. 1B and C). Cytoplasmic $\alpha$-SMA staining was detected in active PSCs which had been cultured for 7 days (Fig. 1D).

PSC promotes chemoresistance to gemcitabine of pancreatic cancer cells. Following treatment with gemcitabine $(100 \mathrm{ng} / \mathrm{ml})$, the growth inhibition rate was $52.3 \pm 12.1$ and $65.1 \pm 16.8 \%$ in the PANC-1 and BxPC-3 cells, respectively. After being cultured with PSC conditioned medium, the inhibition rate significantly decreased to $38.5 \pm 11.6$ and $51.2 \pm 10.9 \%$, respectively (Fig. $2 \mathrm{~A}$ ). The $\mathrm{IC}_{50}$ values were also increased significantly in both pancreatic cancer cell lines (Fig. 2B). Flow cytometric analysis revealed that PSCs promoted the anti-apoptosis ability of the cancer cells. The late apoptosis rate of PANC-1 and the early 

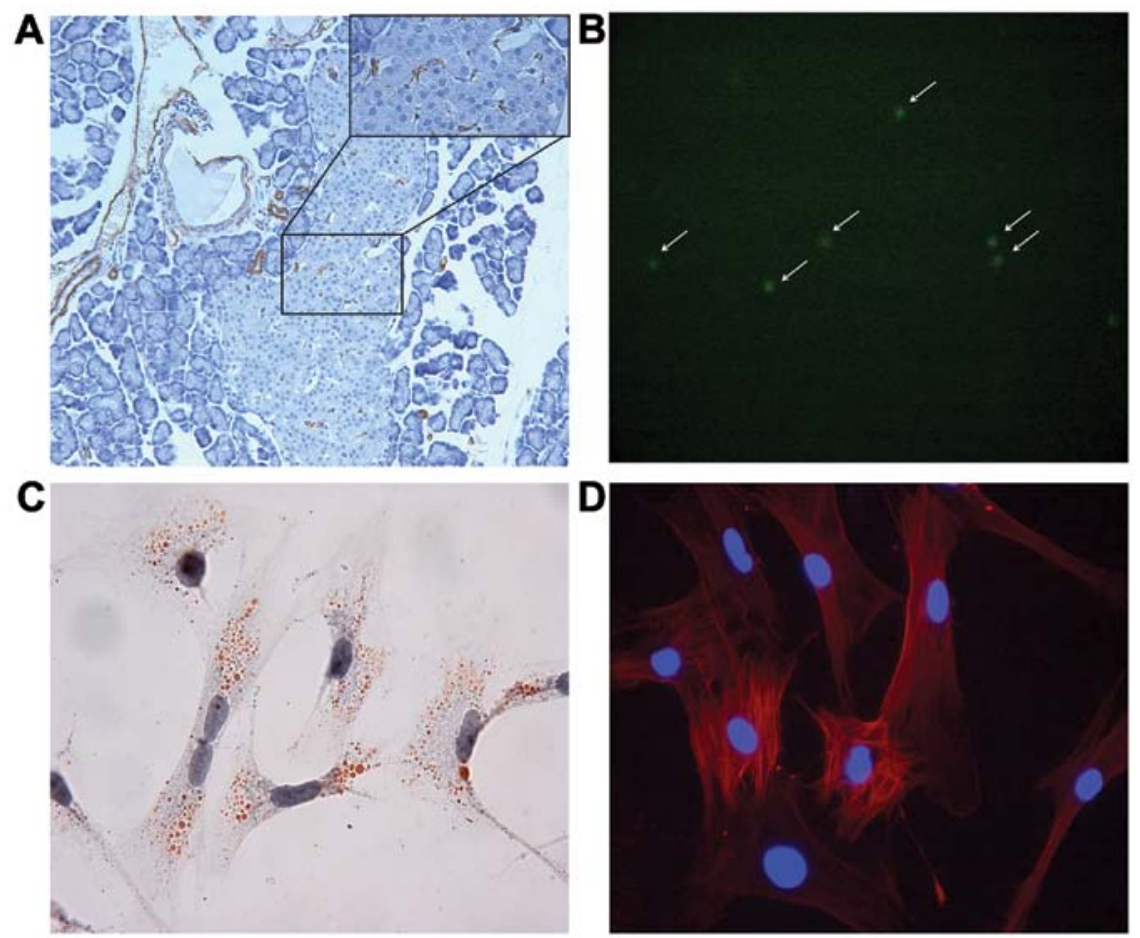

Figure 1. PSCs isolated from normal rat pancreas. (A) Representative sections of $\alpha$-SMA immunostaining of the normal rat pancreas. We found that $\alpha$-SMA positively stained cells were occasionally visible in the islet in normal rat pancreas, which suggests that the PSCs might also exist in the islet and play an important role in maintaining normal structure and function of the islets (magnification, $\mathrm{x} 200$ ). (B) After $24 \mathrm{~h}$ in culture, vitamin A autofluorescence at $328 \mathrm{~nm}$ revealed blue-green fluorescence and indicated the presence of vitamin A in the cytoplasm (arrow, magnification x200). (C) Oil-red O staining indicated perinuclear lipid droplets after $24 \mathrm{~h}$ in culture (magnification x1,000). (D) After seeding for 7 days, positive staining of $\alpha$-SMA was observed in the cytoplasm (magnification, $\mathrm{x} 400$ ). PSCs, pancreatic stellate cells; $\alpha$-SMA, $\alpha$-smooth muscle actin.
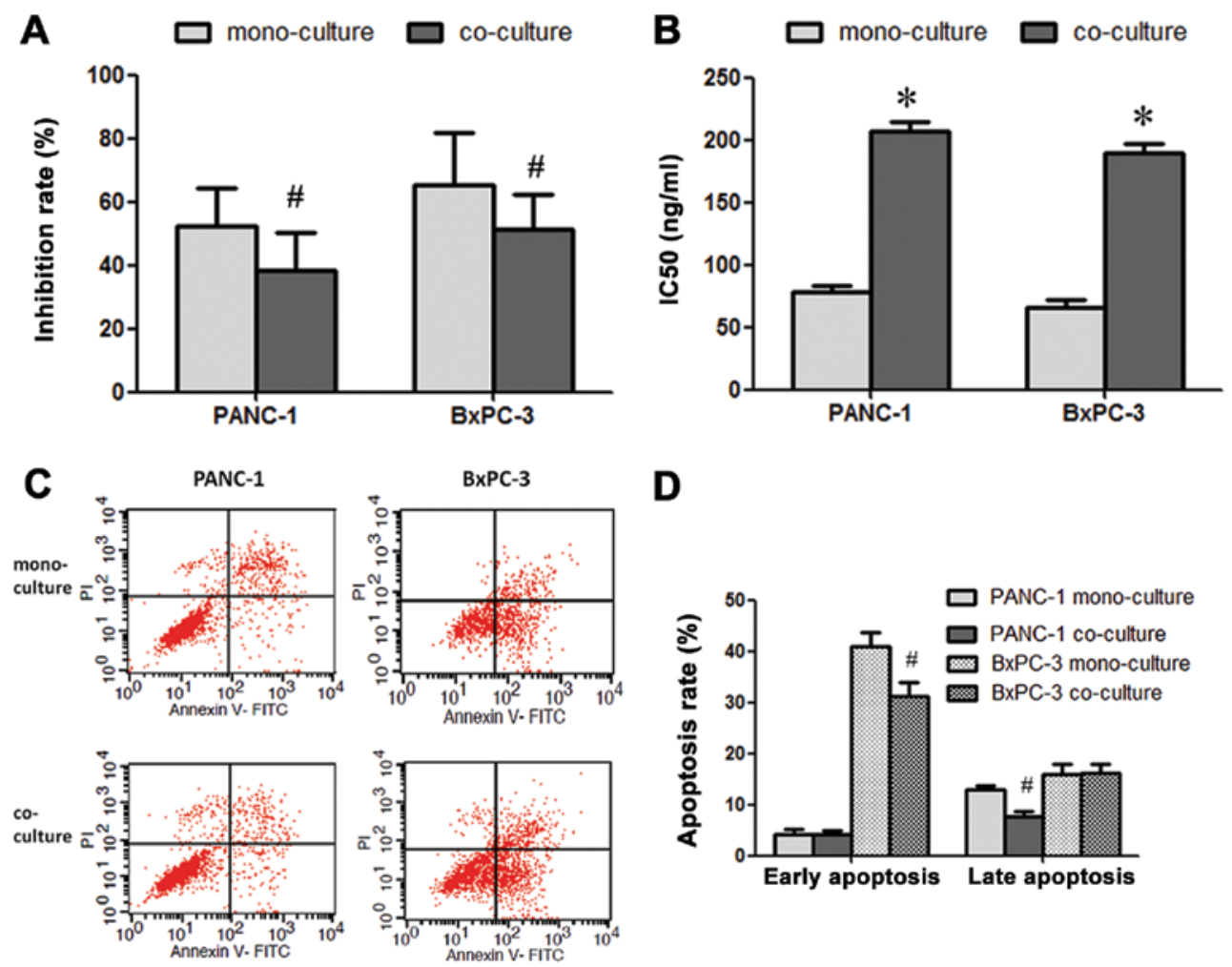

D

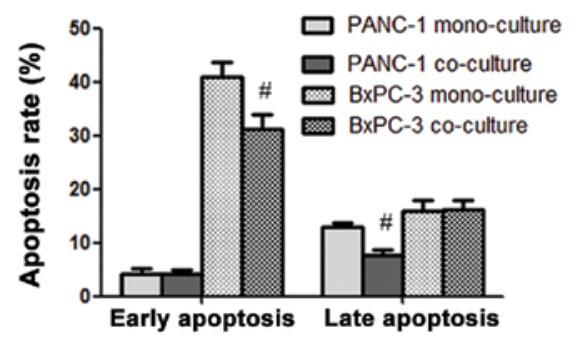

Figure 2. PSCs promote chemoresistance to gemcitabine of pancreatic cancer cells. (A) After being cultured with PSC conditioned medium, the inhibition rate for gemcitabine $(100 \mathrm{ng} / \mathrm{ml})$ was significantly decreased from $52.3 \pm 12.1$ and $65.1 \pm 16.8 \%$ to $38.5 \pm 11.6$ and $51.2 \pm 10.9 \%$ in PANC-1 and BxPC-3 cells, respectively. (B) $\mathrm{The}_{\mathrm{IC}}$ values were also significantly increased from $78.2 \pm 4.8$ to $206.5 \pm 8.2 \mathrm{ng} / \mathrm{ml}$ in the PANC-1 and from $65.4 \pm 6.5$ to $189.6 \pm 8.1 \mathrm{ng} / \mathrm{ml} \mathrm{in}$ the BxPC-3 cells. (C and D) After being co-cultured with PSCs, flow cytometric analysis showed that the late apoptosis rate was significantly decreased from $40.9 \pm 2.8$ to $31.2 \pm 2.6 \%$ in the PANC-1 cells. In the BxPC-3 cells, although the late apoptosis rate did not change significantly before and after co-culture, the early apoptosis rare decreased significantly from $12.8 \pm 0.9$ to $7.8 \pm 1.0 \%$. ${ }^{*} \mathrm{P}<0.01$ vs. the mono-culture; ${ }^{~} \mathrm{P}<0.05$ vs. the mono-culture. PSCs, pancreatic stellate cells. 

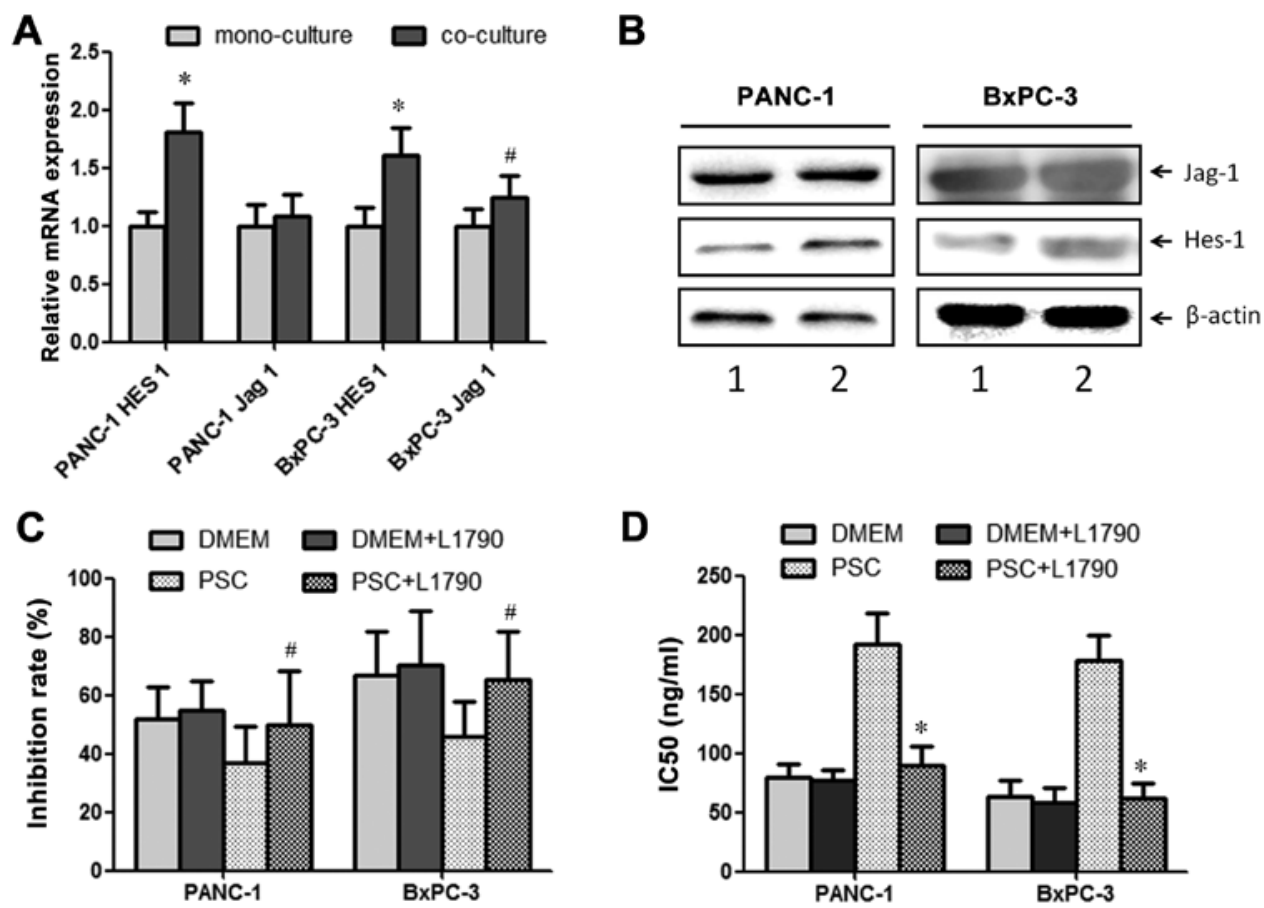

Figure 3. The Notch signaling pathway is involved in the chemoresistance induced by PSCs. (A) RT-PCR analysis showed that expression of Jagged-1 and Hes 1, components of the Notch signaling pathway, was promoted significantly after co-culture with PSCs in both the PANC-1 and BxPC-3 cells. (B) Western blot analysis demonstrated similar results. (C) After introduction of the Notch signaling pathway inhibitor, L1790, the decreased inhibition rate to gemcitabine in the PANC-1 and BxPC-3 was reversed to $49.9 \pm 18.5$ and $65.4 \pm 16.5 \%$, respectively. (D) L1790 had no effect on the $\mathrm{IC}_{50}$ value when cancer cells were mono-cultured. However, when co-cultured with PSCs, the $\mathrm{IC}_{50}$ values were reversed to $89.5 \pm 16.4$ and $61.6 \pm 12.9 \mathrm{ng} / \mathrm{ml}$, respectively. "P<0.01 vs. mono-culture or PSCs; ${ }^{\#} \mathrm{P}<0.05$ vs. PSCs. PSCs, pancreatic stellate cells.
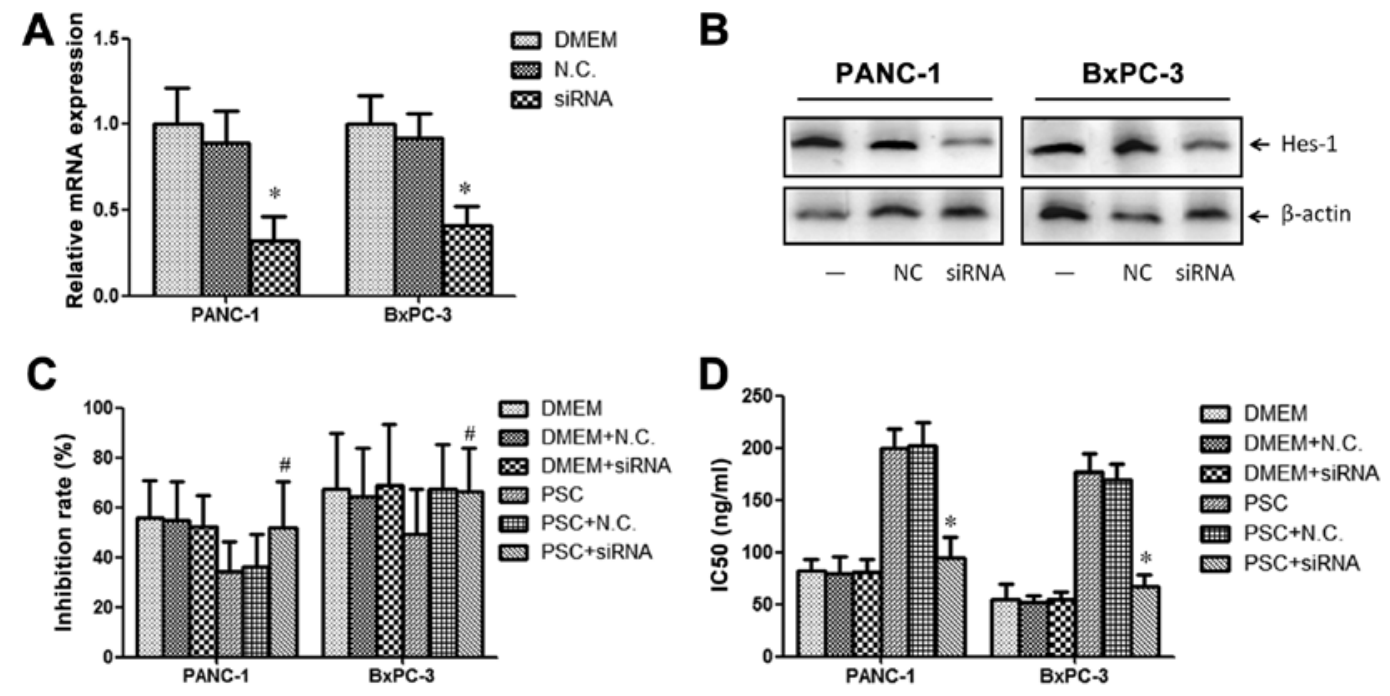

Figure 4. HES 1 is essential for the chemoresistance induced by PSCs. (A and B) siRNA inhibited the expression of Hes 1. After transfection with Hes 1 siRNA, RT-PCR and western blot analysis showed that the expression level of Hes 1 was significantly reduced at $48 \mathrm{~h}$. However, the negative control siRNA had no effect on Hes 1 expression. (C) After transfection with Hes 1 siRNA, the decreased inhibition rate to gemcitabine in the PANC-1 and BxPC-3 cells was reversed to $51.8 \pm 18.6$ and $66.4 \pm 17.6 \%$, respectively. (D) When co-cultured with PSCs, Hes 1 siRNA reversed the $\mathrm{IC}_{50}$ values to $94.5 \pm 20.4 \mathrm{and} 67.5 \pm 11.3 \mathrm{ng} / \mathrm{ml}$. Negative control siRNA had no effect on the $\mathrm{IC}_{50}$ value in the PANC-1 and BxPC-3 cells. ${ }^{*} \mathrm{P}<0.01$ vs. mono-culture or PSCs; ${ }^{*} \mathrm{P}<0.05$ vs. PSCs. PSCs, pancreatic stellate cells.

apoptosis rate of BxPC-3 cells were decreased significantly after co-culture with PSCs (Fig. 2C and D).

Notch signaling pathway is involved in the chemoresistance induced by PSCs. After being co-cultured with PSCs for $48 \mathrm{~h}$, the cancer cells were collected for further analysis. RT-PCR analysis revealed that the expression levels of Jagged 1 and Hes 1, members of the Notch signaling pathway were significantly promoted after co-culture with PSCs in both the PANC-1 and BxPC-3 cell lines (Fig. 3A). Western blot analysis showed similar results (Fig. 3B). In order to determine the role of the Notch signaling pathway in chemoresistance induced 

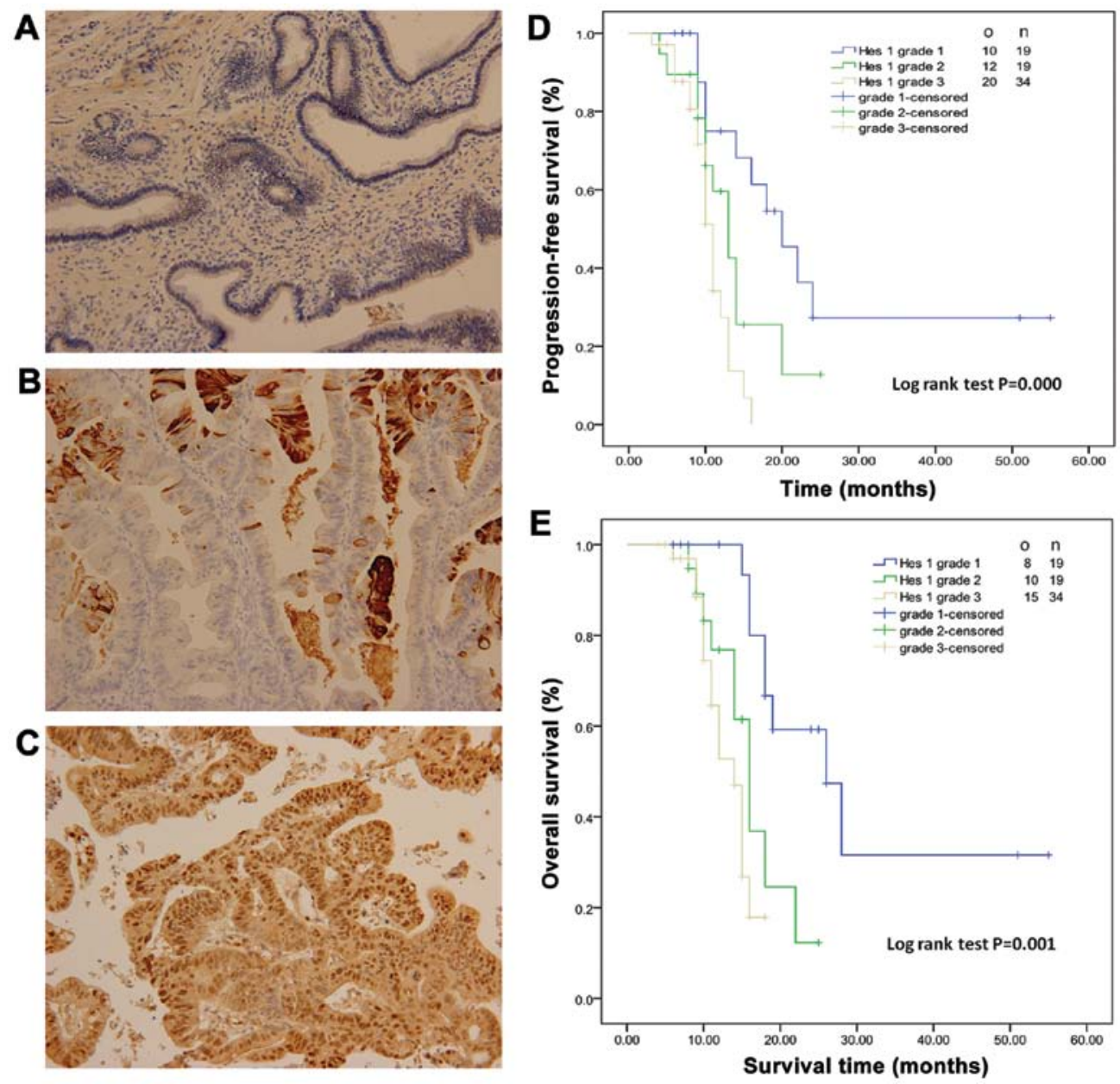

Figure 5. HES 1 expression is associated with poor prognosis in patients with pancreatic cancer. (A-C) Representative staining of Hes 1 in pancreatic cancer patient tumors (magnification, x200). (A) Grade 1, 0-25\% staining; (B) grade 2, 26-50\% staining; (C) grade 3, $>50 \%$ staining. (D) Progression-free survival (PFS) analysis showed that the mean PFS time in grade 1,2 and 3 patients was $26.6 \pm 5.0,13.7 \pm 1.6$ and $10.7 \pm 0.6$ months, respectively. (E) Overall survival (OS) analysis revealed that the mean OS time in grade 1,2 and 3 patients was $31.8 \pm 5.2,16.3 \pm 1.4$ and $13.3 \pm 0.7$ months, respectively. o, observed events; $\mathrm{n}$, number of patients.

Table I. Demographics of the pancreatic cancer patients who underwent resection $(\mathrm{n}=72)$.

\begin{tabular}{lc}
\hline Characteristics & Data \\
\hline Age (years) & $66.5 \pm 11.2$ \\
Gender (male/female) & $45 / 27$ \\
Tumor location (head/body/tail) & $47 / 8 / 17$ \\
Operation (PD/DP) & $47 / 25$ \\
Tumor size (cm) & $3.3 \pm 1.5$ \\
Differentiation (well/moderate/poor) & $7 / 33 / 32$ \\
Lymph node metastasis (yes/no) & $44 / 28$ \\
Perineural infiltration (yes/no) & $29 / 43$ \\
Resection margin (-/+) & $59 / 13$ \\
AJCC/UICC stage (1A/1B/2A/2B) & $6 / 11 / 7 / 48$ \\
\hline
\end{tabular}

$\mathrm{PD}$, pancreaticoduodenectomy including classic whipple procedure and whipple+vascular resection; DP, distal pancreatectomy.

by PSCs, L1790 (5 $\mu \mathrm{M}$, Notch signaling pathway inhibitor) was added to the co-culture system. After introduction of the inhibitor, increased chemoresistance to gemcitabine induced by PSCs was reversed (Fig. 3C). Increased $\mathrm{IC}_{50}$ values for PANC-1 and BxPC-3 cell lines also returned to the levels in the mono-culture (Fig. 3D).

HES 1 is essential for chemoresistance induced by PSCS. To further explore the effect of Hes 1 in the chemoresistance induced by PSCs, we knocked down the expression by siRNA transfection (Fig. 4A and B). After successfully transfection of the Hes 1 siRNA, we found that the effect of PSCs on chemoresistance of PANC-1 and BxPC-3 cells was blocked (Fig. 4C). However, the negative control siRNA did not have any influence on chemoresistance. The effect of PSC on $\mathrm{IC}_{50}$ values for both PANC-1 and BxPC-3 cells was also reversed (Fig. 4D).

HES 1 expression is associated with poor prognosis in patients with pancreatic cancer. Seventy-two patients with pancreatic cancer who underwent resection were included in the present study. Patient demographics are shown in Table I. The majority of patients were male $(62.5 \%, 45 / 72)$ and had cancer located in the head of the pancreas $(65.2 \%, 47 / 72)$. Representative staining of Hes 1 is shown in Fig. 5A-C. Nineteen, 19 and 34 patients had low, moderate and high expression of Hes 1 
Table II. Results of the univariate and multivariate Cox regression analyses for cancer survival.

\begin{tabular}{|c|c|c|c|c|}
\hline \multirow[b]{2}{*}{ Variable } & \multicolumn{2}{|c|}{ Univariate Cox regression } & \multicolumn{2}{|c|}{ Multivariate Cox regression } \\
\hline & $\mathrm{RR}(95 \% \mathrm{CI})$ & P-value & $\mathrm{RR}(95 \% \mathrm{CI})$ & P-value \\
\hline Gender (referent, male) & $1.001(0.321-3.108)$ & 0.115 & $0.981(0.138-5.015)$ & 0.305 \\
\hline Age at diagnosis (referent, $>65$ years) & $2.218(1.234-12.408)$ & $<0.001$ & $2.799(1.194-15.486)$ & $<0.001$ \\
\hline Primary site of tumor (referent, head) & $2.254(0.993-17.134)$ & 0.478 & $3.096(0.256-15.196)$ & 0.641 \\
\hline T stage (referent, T1) & $0.910(0.219-4.952)$ & 0.126 & $0.887(0.326-3.125)$ & 0.213 \\
\hline $\mathrm{N}$ stage (referent, $\mathrm{N} 0$ ) & $0.950(0.275-18.031)$ & 0.271 & $1.010(0.448-16.150)$ & 0.437 \\
\hline Resection margin (referent, positive) & $1.998(0.879-11.258)$ & 0.021 & $2.001(0.714-12.524)$ & 0.019 \\
\hline Gemcitabine therapy (referent, no therapy) & $0.749(0.247-5.867)$ & 0.118 & $0.735(0.312-4.129)$ & 0.069 \\
\hline Hes 1 grade $2+3($ referent, grade 1$)$ & $2.154(1.987-11.212)$ & $<0.001$ & $2.012(1.549-10.214)$ & 0.001 \\
\hline
\end{tabular}

$\mathrm{RR}$, relative risk, $\mathrm{CI}$, confidence interval.

and were scored as grade 1,2 and 3. There was no significant difference among grade 1, 2 and 3 groups (data not shown). The overall survival and progression-free survival time for grade 1 patients were $31.8 \pm 5.2$ and $26.6 \pm 5.0$ months, respectively (Fig. 5D and E). Kaplan-Meier analysis showed that high expression of Hes 1 was associated with shorter overall and progression-free survival (Fig. 5D and E). Hes 1 expression was an independent risk factor for poor prognosis in patients with pancreatic cancer. Cox regression analysis revealed that Hes 1 expression (grade 2 and 3) was an independent risk factor for cancer survival (RR, 2.012, 95\%; CI, 1.549-10.214; $\mathrm{P}=0.001$ ) (Table II).

\section{Discussion}

The role of gemcitabine in the treatment of pancreatic cancer has been established by a series of excellent trials $(6,7,26-30)$. However, the objective response rate remains unsatisfactory $(8,9,31)$. Primary chemoresistance to single-agent gemcitabine occurred in $\sim 34.5 \%$ of metastatic pancreatic cancer patients (8). The addition of cytotoxic and targeted agents to gemcitabine almost invariably provided no significant survival improvement, despite an improvement in response rates in some studies $(7,32,33)$. Stromal cells might play an important role in primary and secondary chemoresistance in cancer patients. As a partner in crime with pancreatic cancer cells, PSCs significantly promote cancer progression in in vivo and in vitro studies (14-16,35). Mounting evidence suggests PSCs are both direct and indirect drivers of pancreatic cancer chemoresistance and spread, and thus elucidation of the underlying mechanisms may potentiate current chemotherapy. Our study demonstrated that PSCs promoted the ability of chemoresistance to gemcitabine in both PANC-1 and BxPC- 3 cells.

While the cause of chemoresistance is multifactorial, three major processes have been largely clarified: i) reduced drug uptake; ii) increased energy-dependent drug efflux; and iii) alterations in cellular capabilities affecting drug cytotoxicity, such as reduced apoptosis and dysregulated drug metabolism (34). Our study also showed that PSCs reduce late apoptosis in PANC-1 and in BxPC-3 cells which may contribute to chemoresistance. In addition, PSCs stimulated the epithelial-mesenchymal transition (EMT) of cancer cells, which resulted in a more chemoresistant phenotype (35). Cancer stem cells are also involved in the chemoresistance induced by PSCs (36). In an in vitro study and in pancreatic cancer patients, another major determinant of pancreatic cancer chemoresistance was the extensive fibrosis produced by PSCs, which resulted in significant intratumoral hypoxia and a self-perpetuating hypoxia-fibrosis cycle. This impaired drug delivery to cancer cells and stimulated their EMT and genetic instability, yielding a more chemoresistant phenotype (34).

More and more evidence has revealed the fact that the Notch signaling pathway may be a potential target for reversing the chemoresistance of pancreatic cancer. Gungor et al showed that Midkine-Notch-2 interaction activated Notch signaling, induced EMT, upregulated Hes 1 and increased chemoresistance (37). Wang et al demonstrated that in gemcitabine-resistant pancreatic cancer cells, the Notch signaling pathway was overactivated with Notch-2 and Jagged-1 overexpression (38). Kang et al showed that Notch ligand Delta-like 4 (DLL4) induced impaired chemo-drug delivery and enhanced chemoresistance in pancreatic cancer in vivo. Overactivation of the DLL4/Notch pathway enhanced the phenotype of EMT and cancer stem cells, and induced multi-chemoresistance in vitro (39). Our study also demonstrated that PSCs promoted Hes 1 expression and overactivated the Notch signaling pathway. L1790 (Notch signaling pathway inhibitor) and Hes 1 siRNA reversed the chemoresistance induced by PSCs. These results provide molecular evidence showing that Hes 1 is essential for the chemoresistance induced by PSCs.

In view of the Notch signaling pathway in the development of pancreatic cancer, it is not surprising that the Notch expression status is associated with the prognosis of pancreatic cancer patients. We found that Hes 1 high expression is a biomarker for poor prognosis in pancreatic adenocarcinoma.

In conclusion, our results suggest that PSCs induce Hes 1 expression and promote chemoresistance in pancreatic cancer. Hes 1 is an effective prognostic factor and is significantly associated with prognosis of pancreatic cancer patients. Therapy targeting the Notch signaling pathway may reverse chemoresistance and improve survival in patients with advanced pancreatic cancer. 


\section{References}

1. Wray CJ, Ahmad SA, Matthews JB and Lowy AM: Surgery for pancreatic cancer: recent controversies and current practice. Gastroenterology 128: 1626-1641, 2005.

2. Cameron JL, Riall TS, Coleman J and Belcher KA: One thousand consecutive pancreaticoduodenectomies. Ann Surg 244: 10-15, 2006.

3. Raman SP, Horton KM, Cameron JL and Fishman EK: CT after pancreaticoduodenectomy: spectrum of normal findings and complications. AJR Am J Roentgenol 201: 2-13, 2013.

4. Siegel R, Ma J, Zou Z and Jemal A: Cancer statistics, 2014. CA Cancer J Clin 64: 9-29, 2014.

5. Loehrer PJ Sr, Feng Y, Cardenes H, et al: Gemcitabine alone versus gemcitabine plus radiotherapy in patients with locally advanced pancreatic cancer: an Eastern Cooperative Oncology Group trial. J Clin Oncol 29: 4105-4112, 2011.

6. Colucci G, Labianca R, Di Costanzo F, et al: Randomized phase III trial of gemcitabine plus cisplatin compared with single-agent gemcitabine as first-line treatment of patients with advanced pancreatic cancer: the GIP-1 study. J Clin Oncol 28: 1645-1651, 2010.

7. Cunningham D, Chau I, Stocken DD, et al: Phase III randomized comparison of gemcitabine versus gemcitabine plus capecitabine in patients with advanced pancreatic cancer. J Clin Oncol 27: 5513-5518, 2009

8. Conroy T, Desseigne F, Ychou M, et al: FOLFIRINOX versus gemcitabine for metastatic pancreatic cancer. N Engl J Med 364 1817-1825, 2011.

9. Von Hoff DD, Ervin T, Arena FP, et al: Increased survival in pancreatic cancer with nab-paclitaxel plus gemcitabine. N Engl J Med 369: 1691-1703, 2013.

10. Bachem MG, Schneider E, Gross H, et al: Identification, culture and characterization of pancreatic stellate cells in rats and humans. Gastroenterology 115: 421-432, 1998.

11. Apte MV, Haber PS, Applegate TL, et al: Periacinar stellate shaped cells in rat pancreas: identification, isolation, and culture Gut 43: 128-133, 1998.

12. Fujita H, Ohuchida K, Mizumoto K, et al: Tumor-stromal interactions with direct cell contacts enhance proliferation of human pancreatic carcinoma cells. Cancer Sci 100: 2309-2317, 2009.

13. Vonlaufen A, Joshi S, Qu C, et al: Pancreatic stellate cells: partners in crime with pancreatic cancer cells. Cancer Res 68 2085-2093, 2008.

14. Hwang RF, Moore T, Arumugam T, et al: Cancer-associated stromal fibroblasts promote pancreatic tumor progression. Cancer Res 68: 918-926, 2008.

15. Mantoni TS, Lunardi S, Al-Assar O, Masamune A and Brunner TB: Pancreatic stellate cells radioprotect pancreatic cancer cells through $\beta 1$-integrin signaling. Cancer Res 71 3453-3458, 2011.

16. Farrow B, Berger DH and Rowley D: Tumor-derived pancreatic stellate cells promote pancreatic cancer cell invasion through release of thrombospondin-2. J Surg Res 156: 155-160, 2009.

17. Greenwald I and Kovall R: Notch signaling: genetics and structure. WormBook: 1-28, 2013

18. Nicolas M, Wolfer A, Raj K, et al: Notch1 functions as a tumor suppressor in mouse skin. Nat Genet 33: 416-421, 2003.

19. Radtke F and RajK: The role of Notch in tumorigenesis: oncogene or tumour suppressor? Nat Rev Cancer 3: 756-767, 2003.

20. Mann CD, Bastianpillai C, Neal CP, et al: Notch3 and HEY-1 as prognostic biomarkers in pancreatic adenocarcinoma. PLoS One 7: e51119, 2012

21. Yabuuchi S,Pai SG, Campbell NR, et al: Notch signaling pathway targeted therapy suppresses tumor progression and metastatic spread in pancreatic cancer. Cancer Lett 335: 41-51, 2013
22. Mizuma M, Rasheed ZA, Yabuuchi S, et al: The gamma secretase inhibitor MRK-003 attenuates pancreatic cancer growth in preclinical models. Mol Cancer Ther 11: 1999-2009, 2012.

23. Cook N, Frese KK, Bapiro TE, et al: Gamma secretase inhibition promotes hypoxic necrosis in mouse pancreatic ductal adenocarcinoma. J Exp Med 209: 437-444, 2012.

24. Schott AF, Landis MD, Dontu G, et al: Preclinical and clinical studies of gamma secretase inhibitors with docetaxel on human breast tumors. Clin Cancer Res 19: 1512-1524, 2013.

25. Yamada S, Fuchs BC, Fujii T, et al: Epithelial-to-mesenchymal transition predicts prognosis of pancreatic cancer. Surgery 154 : 946-954, 2013.

26. Van Cutsem E, van de Velde H, Karasek P, et al: Phase III trial of gemcitabine plus tipifarnib compared with gemcitabine plus placebo in advanced pancreatic cancer. J Clin Oncol 22: 1430-1438, 2004.

27. Abou-Alfa GK, Letourneau R, Harker G, et al: Randomized phase III study of exatecan and gemcitabine compared with gemcitabine alone in untreated advanced pancreatic cancer. J Clin Oncol 24: 4441-4447, 2006

28. Heinemann V, Quietzsch D, Gieseler F, et al: Randomized phase III trial of gemcitabine plus cisplatin compared with gemcitabine alone in advanced pancreatic cancer. J Clin Oncol 24: 3946-3952, 2006.

29. Van Cutsem E, Vervenne WL, Bennouna J, et al: Phase III trial of bevacizumab in combination with gemcitabine and erlotinib in patients with metastatic pancreatic cancer. J Clin Oncol 27: 2231-2237, 2009

30. Ueno H, Ioka T, Ikeda M, et al: Randomized phase III study of gemcitabine plus S-1, S-1 alone, or gemcitabine alone in patients with locally advanced and metastatic pancreatic cancer in Japan and Taiwan: GEST study. J Clin Oncol 31: 1640-1648, 2013.

31. Vaccaro V, Sperduti I and Milella M: FOLFIRINOX versus gemcitabine for metastatic pancreatic cancer. N Engl J Med 365: 768-769, 2011.

32. Louvet C, Labianca R, Hammel P, et al: Gemcitabine in combination with oxaliplatin compared with gemcitabine alone in locally advanced or metastatic pancreatic cancer: results of a GERCOR and GISCAD phase III trial. J Clin Oncol 23: 3509-3516, 2005.

33. Colucci G, Giuliani F, Gebbia V, et al: Gemcitabine alone or with cisplatin for the treatment of patients with locally advanced and/or metastatic pancreatic carcinoma: a prospective, randomized phase III study of the Gruppo Oncologia dell'Italia Meridionale. Cancer 94: 902-910, 2002.

34. McCarroll JA, Naim S, Sharbeen G, et al: Role of pancreatic stellate cells in chemoresistance in pancreatic cancer. Front Physiol 5: 141, 2014.

35. Kikuta K, Masamune A, Watanabe T, et al: Pancreatic stellate cells promote epithelial-mesenchymal transition in pancreatic cancer cells. Biochem Biophys Res Commun 403: 380-384, 2010.

36. Izumiya M, Kabashima A, Higuchi $\mathrm{H}$, et al: Chemoresistance is associated with cancer stem cell-like properties and epithelial-to-mesenchymal transition in pancreatic cancer cells. Anticancer Res 32: 3847-3853, 2012.

37. Gungor C, Zander H, Effenberger KE, et al: Notch signaling activated by replication stress-induced expression of midkine drives epithelial-mesenchymal transition and chemoresistance in pancreatic cancer. Cancer Res 71: 5009-5019, 2011

38. Wang Z, Li Y, Kong D, et al: Acquisition of epithelial-mesenchymal transition phenotype of gemcitabine-resistant pancreatic cancer cells is linked with activation of the notch signaling pathway. Cancer Res 69: 2400-2407, 2009.

39. Kang M, Jiang B, Xu B, et al: Delta like ligand 4 induces impaired chemo-drug delivery and enhanced chemoresistance in pancreatic cancer. Cancer Lett 330: 11-21, 2013. 